\title{
Erratum to: Pregnancy outcome among partners of male patients receiving imatinib, dasatinib or nilotinib in chronic myeloid leukemia: reports collected by the French network pharmacovigilance centers
}

Patrick Carlier $^{1} \cdot$ Maritza Markarian $^{1} \cdot$ Nathalie Bernard $^{2} \cdot$ Laurence Lagarce $^{3}$.

Anne Dautriche $^{4} \cdot$ Johanna Béné $^{5} \cdot$ Nathalie Fouilhe Sam-Lai ${ }^{6} \cdot$ Pirayeh Eftekhari $^{1}$

Published online: 3 March 2017

(C) Springer-Verlag Berlin Heidelberg 2017

Erratum to: Arch Gynecol Obstet (2017)

295:269-271

DOI 10.1007/s00404-016-4262-z

Unfortunately, a co-author's name was not listed in the original publication. The missing author name and affiliation is given below:

Patrick Carlier ${ }^{1}$, Maritza Markarian ${ }^{1}$, Nathalie Bernard ${ }^{2}$, Laurence Lagarce ${ }^{3}$, Anne Dautriche ${ }^{4}$, Johanna Béné ${ }^{5}$, Nathalie Fouilhe Sam-Lai ${ }^{6}$, Pirayeh Eftekhari ${ }^{1}$.

The online version of the original article can be found under doi:10.1007/s00404-016-4262-z.

Patrick Carlier

pharmacovigilance.fwd.lrb@aphp.fr

1 Centre régional de Pharmacovigilance, Hôpital FernandWidal, AP-HP, Paris, France

2 Centre régional de Pharmacovigilance, Hospices Civils de Lyon, Lyon, France

3 Centre régional de Pharmacovigilance, CHRU Angers, Angers, France

4 Centre régional de Pharmacovigilance, CHU Dijon, Dijon, France

5 Centre régional de Pharmacovigilance du Nord Pas de Calais, CHU Lille, 59000 Lille, France

6 Centre régional de Pharmacovigilance, CHU Grenoble Alpes, Grenoble, France 